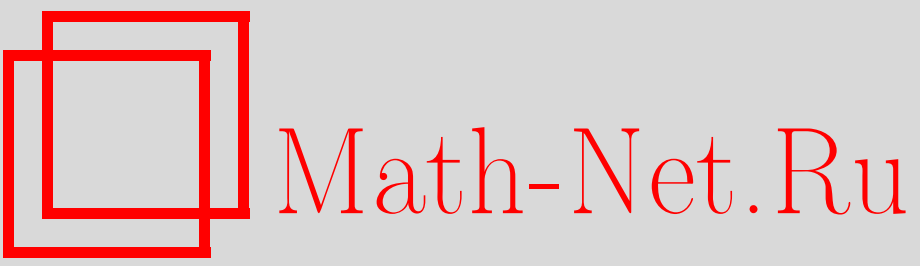

А. М. Вершик, А. Ю. Окуньков, Индуктивный способ изложения теории представлений симметрических групп, УМН, 1996, том 51, выпуск 2, 153-154

DOI: https://doi.org/10.4213/rm952

Использование Общероссийского математического портала Math-Net.Ru подразумевает, что вы прочитали и согласны с пользовательским соглашением

http://www . mathnet.ru/rus/agreement

Параметры загрузки:

IP : 107.22 .136 .117

26 апреля 2023 г., 15:53:26 


\title{
ИНДУКТИВНЫЙ СПОСОБ ИЗЛОЖЕНИЯ ТЕОРИИ ПРЕДСТАВЛЕНИЙ СИММЕТРИЧЕСКИХ ГРУПП
}

\author{
А. М. ВеРшик, А. Ю. ОкУньков
}

1. Существующие до настоящего времени способы построения теории представлений симметрических групп, берущие начало от работ А. Юнга, И. Шура, Г. Фробениуса и др., используют диаграммы Юнга и их комбинаторику. Неслучайный характер появления диаграмм в этой теории становится ясным лишь в самом конце построений, когда выясняется, что ветвление представлений $\mathfrak{S}_{n} \mid \mathfrak{S}_{n-1}$ совпадает с ветвлением диаграмм Юнга с $n$ клетками по диаграммам с $n-1$ клеткой. Недостатки традиционного способа (переходящего из книги в книгу уже около 100 лет) очевидны: во-первых, хотелось бы оправдать появление диаграмм apriori (a не aposteriori), во-вторых, существующая техника (см. [1]) не использует кокстеровость групп и не переносится на общие группы Кокстера-Вейля, в-третьих, отсутствует индуктивность построений - не используется то, что $\mathfrak{S}_{n}$ образуют серию. В [2] показано, что из кокстеровских соотношений и дистрибутивности ветвления можно вывести само правило ветвления и найти матрицы представления (ортогональную форму Юнга). В этой работе мы, наконец, избавляемся от всех дополнительных ограничений: только из соотношений Кокстера, используя так называемые образующие Мерфи-Юциса (МЮ), находим спектр алгебры Гельфанда-Цетлина групп $\mathfrak{S}_{n}$ и, тем самым, реализуем план построения теории представлений групп $\mathfrak{S}_{n}$, намеченный в [2], [3], открывая возможность для переноса метода на все группы Кокстера и локальные алгебры [3].

2. Группа $\mathfrak{S}_{n}$ определяется образуюшими $\sigma_{1}, \sigma_{2}, \ldots, \sigma_{n-1}$ и соотношениями $\sigma_{i}^{2}=1$, $\sigma_{i} \sigma_{j}=\sigma_{j} \sigma_{i},|i-j|>1,\left(\sigma_{i} \sigma_{i+1}\right)^{3}=1$. Алгебра Гельфанда-Цетлина $G Z_{n}$ есть подалгебра групповой алгебры $\mathbb{C}\left(\mathfrak{S}_{n}\right)$, порожденная центрами всех подалгебр $\mathbb{C}\left(\mathfrak{S}_{k}\right), k=1, \ldots, n$.

Теорема. Алгебра $G Z_{n}$ порождается образующими $М Ю$ [4], [5]: $X_{k}, k=1, \ldots, n$, $X_{k}=\sum_{i=1}^{k-1}(i, k), X_{1}=0$, и является максимальной коммутативной подалгеброй в $\mathbb{C}\left(\mathfrak{S}_{n}\right) . \overline{\Pi р и ~ ә т о м ~ в ы п о л н е н ы ~ с о о т н о ш е н и я ~}$

$$
\sigma_{i} X_{j}=X_{j} \sigma_{i}, \quad|i-j|>1, \quad \sigma_{i} X_{i}+1=X_{i+1} \sigma_{i}, \quad i=1, \ldots, n .
$$

Заметим, что определение $G Z_{n}$ имеет смысл для любой локально-полупростой и любой локальной алгебры, а не только для классических алгебр Ли, как это трактовалось до сих пор, см. [2], [6].

В каждом неприводимом комплексном представлении $\pi$ группы $\mathfrak{S}_{n}$ существует базис $V^{\pi}$ из собственных векторов алгебры $G Z_{n}$, и, если $v \in V^{\pi}$, то спектральным вектором $s^{\pi}(v)=$ $\left(s_{1}(v), \ldots, s_{n}(v)\right)$ назовем вектор из собственных чисел: $X_{i} v=s_{i}(v) v, i=1, \ldots, n$. Легко доказать, что спектр $G Z_{n}$ как коммутативной алгебры есть $\operatorname{Spec} G Z_{n}=\left\{s^{\pi}(v): v \in V^{\pi}, \pi \in \widehat{\mathfrak{S}}_{n}\right\}$. Для построения теории представлений $\mathfrak{S}_{n}$ (как и любой локальной алгебры) достаточно описать а) спектр алгебры $G Z_{n}$; б) разбиение спектра на части, входящие в одно и то же неприводимое представление $\pi \in \widehat{\mathfrak{S}}_{n} ;$ в) матричные элементы для образующих.

3. Содержательным вектором называется целочисленный вектор $a=\left(a_{1}=0, a_{2}, \ldots, a_{n}\right)$ $\in \mathbb{Z}^{n}$, удовлетворяющий условиям: 1) $\left.\forall k<n \exists i<k:\left|a_{i}-a_{k}\right|=1,2\right)$ если $a_{k}=a_{k+s}=a$, то $\exists i, j<s: a_{k+i}=a+1, a_{k+j}=a-1$. Множество содержательных векторов обозначим $\operatorname{Cont}_{n} \subset \mathbb{Z}^{n}$.

Tеорема. Spec $G Z_{n} \cong \operatorname{Cont}_{n}$, причем образующие $X_{i}$ при этом изоморфизме переходят в координатнье функциональ на $\mathbb{R}^{n}$.

Работа выполнена при финансовой поддержке Российского фонда фундаментальных исследований (грант № 94-01-00921). 
ДокаЗАтельство. Наиболее важно включение $\operatorname{Spec} G Z_{n} \subset \operatorname{Cont}_{n}$. Для $n=2$ это очевидно. Пусть включение доказано для $n$ и $a \in \operatorname{Spec} G Z_{n+1}$. Тогда существуют представление $\pi \in \widehat{\mathfrak{S}}_{n+1}$ группы $\mathfrak{S}_{n+1}$ и вектор $v \in V^{\pi}$, для которого $X_{i} v=a_{i} v, i=1, \ldots, n+1$. Из соотношений для $X_{i}$ и $\sigma_{i}$ вытекает, что подпространство $L_{n}$, натянутое на векторы $v, \sigma_{n} v$, является собственным для $G Z_{n+1}$. Если $\operatorname{dim} L_{n}=2$, то существует вектор $v^{\prime} \in V^{\pi} \cap L_{n}$, для которого $X_{n} v^{\prime}=a_{n+1} v^{\prime}, X_{n+1} v^{\prime}=a_{n} v^{\prime}$, тем самым, по индукционному предположению оба вектора $\left(a_{1}, \ldots, a_{n-1}, a_{n+1}\right),\left(a_{1}, \ldots, a_{n-1}, a_{n}\right)$ лежат в Cont $n$, а отсюда, используя соотношения для $\sigma_{n}, \sigma_{n+1}$, легко вывести, что $\left(a_{1}, \ldots, a_{n-1}, a_{n}, a_{n+1}\right) \in \operatorname{Cont}_{n+1}$. Если же $\operatorname{dim} L_{n}=1$, то $\left|a_{n}-a_{n+1}\right|=1$, и нужный факт следует из прямой проверки определения содержательности.

4. Пусть $\lambda$ - диаграмма Юнга с $n$ клетками. Содержсанием клетки $\alpha \in \lambda$ называется число $c(\alpha)=i-j$, где $i, j-$ координаты клетки. Понятие содержания известно из классических работ.

Теорема. Пусть $t$ - таблица Юнга с п клетками $u\left\{t_{i}\right\}_{i=1}^{n}-$ последовательность содержаний клеток таблицы $t$. Соответствие $t \rightarrow\left\{t_{i}\right\}_{i=1}^{n}$ есть биекция межсду множеством таблии с п клетками и множеством Cont $n$. Множество векторов из $\operatorname{Cont}_{n}$, отвечающих таблицам с данной диаграммой, есть множество векторов $c$ одинаковым составом координат. Это множество отвечает части спектра алгебры $G Z_{n}$, входящей в одно неприводимое представление $\mathfrak{S}_{n}$.

Нахождение явных матриц представления для образующих $\sigma_{i}, i=1, \ldots, n$ (ортогоналњная или полунормальная форма Юнга) уже не представляет труда и фактически не раз проводилось [2], [4], [5].

\section{СПИСОК ЛИТЕРАТУРЫ}

[1] Джеймс Г. Теория представлений симметрических групп. М.: Мир, 1982. [2] Vershik A. // Banach Center Publ. 1990. V. 26. Part 2. P. 467-473. [3] Vershik A. // Selecta Math. 1992. V. 1. № 2. P. 159-180. [4] Jucys A. // Reports Math. Phis. 1974. V. 5. P. 107-112. [5] Murphy G. // J. Algebra. 1981. V. 69. P. 287-291. [6] Вершик А., Керов С. // Итоги науки и техники. Соврем. проблемы матем. Новейшие достижения. Т. 26. М.: ВИНИТИ, 1985. C. $3-56$.

С.-Петербургское отделение

Математического института им. В.А. Стеклова РАН;

Принято редколлегией Московский государственный университет им. М. В. Ломоносова 